\title{
Technical Note
}

\section{Arthroscopic Management of Locked Posterior Shoulder Dislocations}

\author{
George W. Byram III, M.D., Adam C. Field, B.S., and Larry D. Field, M.D.
}

\begin{abstract}
Locked posterior shoulder dislocations occur relatively uncommonly but pose aunique array of challenges for the treating surgeon. Posterior labral detachments and capsular damage along with reverse Hill-Sachs lesions are commonly present in these patients and must often be addressed at the time of surgical intervention. Multiple open and arthroscopic procedures that address these pathologic lesions have been described. Arthroscopic shoulder stabilization for patients with locked posterior shoulder dislocations for whom significant posterior instability and subluxation persist following closed reduction is described. A specific patient who underwent both arthroscopic posterior stabilization and supplemental advancement of the subscapularis into the reverse Hill-Sachs defect serves as a case example of this technique.
\end{abstract}

$\mathbf{L}$ ocked posterior shoulder dislocations represent a relatively uncommon subset of dislocations, but management of these injuries poses significant challenges for the treating physician. Unfortunately, failure to identify this condition at the time of initial presentation using clinical and radiographic evaluation results in significant delays in the treatment of this complex condition that can compromise outcomes. In fact, failure of early identification and appropriate treatment of locked posterior shoulder dislocations can have serious consequences and contribute to recurrent instability. ${ }^{1-3}$ The reverse Hill-Sachs lesion, an impaction fracture of the anteromedial humeral head, is a bony lesion found in shoulders with posterior instability following posterior glenohumeral joint dislocations. This defect, concomitant posterior capsulolabral injuries, posterior bony glenoid lesions, and posterior capsular attenuation

From the Mississippi Sports Medicine and Orthopaedic Center, Jackson, Mississippi, U.S.A.

The authors report the following potential conflicts of interest or sources of funding: L.D.F. receives support from AANA, Smith $\theta$ Nephew, Arthrex, and Mitek. Full ICMJE author disclosure forms are available for this article online, as supplementary material.

Received February 20, 2018; accepted May 25, 2018

Address correspondence to Dr. Larry D. Field, M.D., Mississippi Sports Medicine and Orthopaedic Center, 1325, Jackson, MS 39202, U.S.A. E-mail: lfield@msmoc.com

(C) 2018 by the Arthroscopy Association of North America. Published by Elsevier. This is an open access article under the CC BY-NC-ND license (http:// creativecommons.org/licenses/by-nc-nd/4.0/).

$2212-6287 / 18255$

https://doi.org/10.1016/j.eats.2018.05.008 have all been described and have been shown to predispose to recurrent posterior shoulder instability. ${ }^{1-5}$

Multiple open procedures have been described to address the reverse Hill-Sachs lesion, but with the improved capabilities of minimally invasive techniques, arthroscopic management of these complex conditions is often possible. Potential benefits of arthroscopic treatment of a locked posterior dislocation include the opportunity to address not only the posterior labral, capsular, and glenoid damage in a minimally invasive manner but also to arthroscopically access and treat the reverse Hill-Sachs lesion, when indicated. This is due to the unique ability of arthroscopic intervention to access the entire glenohumeral joint while minimizing the iatrogenic insult inherent in an open surgical approach (Table 1). Arthroscopic treatment also obviates the requirement to detach the subscapularis tendon, a key stabilizer resisting posterior translation and contributing very significantly to joint stability and function. ${ }^{5-10}$ The arthroscopic technique described and illustrated here is accomplished using standard arthroscopic portals and implants. The subscapularis advancement into the reverse Hill-Sachs defect is technically similar to the arthroscopic remplissage technique used for the treatment of the more commonly encountered posterolateral Hill-Sachs lesion seen in anterior shoulder instability cases.

\section{Indications}

Surgical intervention is recommended in an active, young healthy patient who presents with a subacute locked posterior glenohumeral dislocation. Arthroscopic 
Table 1. Advantages and Disadvantages of Arthroscopic Reverse Remplissage

\begin{tabular}{ll}
\hline \multicolumn{1}{c}{ Advantages } & \multicolumn{1}{c}{ Disadvantages } \\
\hline Minimally invasive. & Technically demanding. \\
Standard arthroscopic setup and familiar portals. & Not easily converted into a bony procedure. \\
$\begin{array}{l}\text { Ability to address coinciding joint pathology. } \\
\text { Recentering of humeral head on glenoid directly visualized with anterosuperior } \\
\text { portal. }\end{array}$ & Dependence on subscapularis tissue quality. \\
No need for specialized implants or grafts. & \\
\hline
\end{tabular}

surgery is indicated in the setting of continued shoulder pain, persistent weakness, and significant motion loss. The radiographic series performed at the time of clinical assessment demonstrates a locked posterior shoulder dislocation with an associated reverse Hills-Sachs lesion (Fig 1 A, B, and C). Closed versus open glenohumeral joint reduction with either arthroscopic or open stabilization as necessary is based on an intraoperative assessment of stability.

\section{Surgical Technique}

Following the induction of general anesthesia, the patient is placed in the beach chair position. Successful reduction of the glenohumeral joint is then performed and confirmed fluoroscopically. Following joint relocation, an intraoperative assessment of stability is performed, evaluating for persistent posterior instability or spontaneous redislocation with minimal posteriorly directed force. A high degree of posterior instability may persist following closed reduction, thus arthroscopic assessment and stabilization are indicated. After establishing standard posterior and anterior portals, a thorough diagnostic arthroscopic assessment is performed. Next, using spinal needle localization, an anterosuperior viewing portal is established just anterior to the biceps tendon. While viewing with a $30^{\circ}$ arthroscope through the anterosuperior portal, a large posterior capsular rent associated with significant posterior humeral head subluxation is identified (Fig 2). Two $3 \mathrm{~mm}$ biocomposite suture anchors (Arthrex, Naples, FL) are inserted into the posterior glenoid rim adjacent to the articular surface, and a $60^{\circ}$ retrograde suture retriever (IDEAL suture passer, Depuy Mitek, Raynham, MA) is used to pass sutures through both the medial and lateral aspects of this capsular split. These sutures are then tied in a mattress fashion, reapproximating the capsular split and significantly reducing inferior and posterior capsular volume (Fig 3). However, while the degree of overall glenohumeral joint alignment is improved following the posterior capsular procedure, significant residual posterior humeral head subluxation persists. Glenohumeral joint stability is assessed intraoperatively following arthroscopic posterior stabilization and demonstrates that, while posterior translation is notably decreased, excessive posterior translation and subluxation remain. As a consequence, the surgeons elect to also advance the subscapularis tendon into the reverse Hills-Sachs defect in an effort to improve stability and overall glenohumeral joint alignment.

While viewing from the anterosuperior portal, an $8.75-\mathrm{mm}$ threaded cannula (Arthrex) is inserted through the anterior working portal. The reverse HillSachs lesion is identified, and this impacted bony bed is debrided using an arthroscopic shaver to remove any soft tissue and to stimulate this bony surface (Fig 4) in preparation for advancement of the subscapularis tendon into this defect. Importantly, the arthroscopic shaver is also used to excise the central portion of the
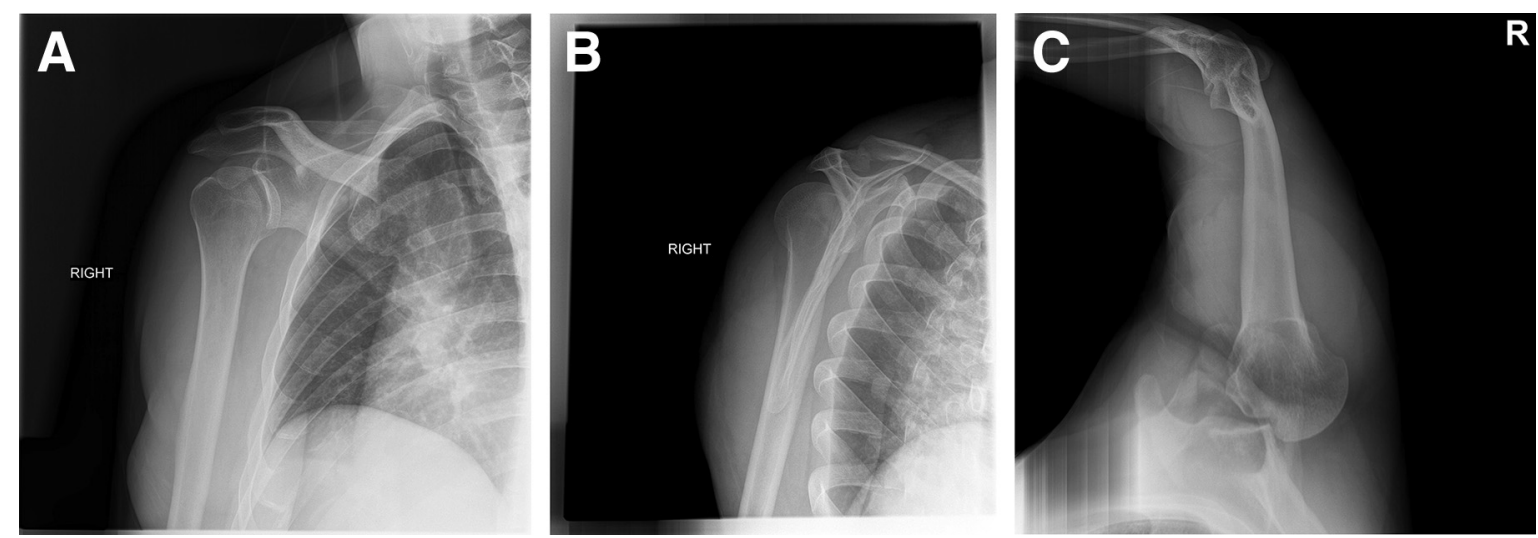

Fig 1. (A) Anteroposterior view of the right shoulder showing a posterior glenohumeral dislocation, dislocation. (B) Scapular Y view of the right shoulder showing posterior glenohumeral dislocation, dislocation. (C) Axillary view of the right shoulder showing locked posterior glenohumeral dislocation. Engaging reverse Hills-Sachs lesion is evident. 


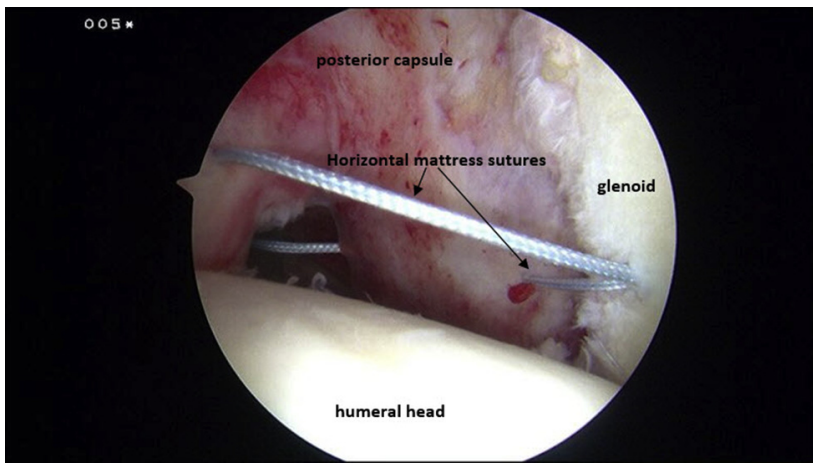

Fig 2. Arthroscopic view of the right shoulder in the beach chair position with a $30^{\circ}$ arthroscope in the anterosuperior portal. A large posterior capsular rent and posterior humeral subluxation is appreciated. One of 2 biocomposite suture anchors (Arthrex) is then placed on the posterior glenoid rim. A retrograde suture retriever (IDEAL suture passer) is used to retrieve sutures through both the medial and lateral aspects of the capsular split. This suture is then tied in a mattress fashion.

rotator interval tissue to allow for adequate access to and visualization of the anterior surface to the subscapularis tendon to facilitate suture passage across the subscapularis tendon as well as knot tying. A 5.5-mm triple-loaded biocomposite suture anchor (Smith \& Nephew, Andover, MA) is then inserted into the central area of the reverse Hill-Sachs lesion bony defect. The arthroscope is then transferred to the posterior portal to visualize suture retrieval across the subscapularis tendon (Fig 5). The $60^{\circ}$ retrograde suture retriever is sequentially passed through the subscapularis tendon and used to retrieve sutures. The suture anchor sutures are passed in a combined horizontal mattress and simple pattern resulting in a "rip-stop" suture configuration (Fig 6). This rip-stop suture construct pattern requires

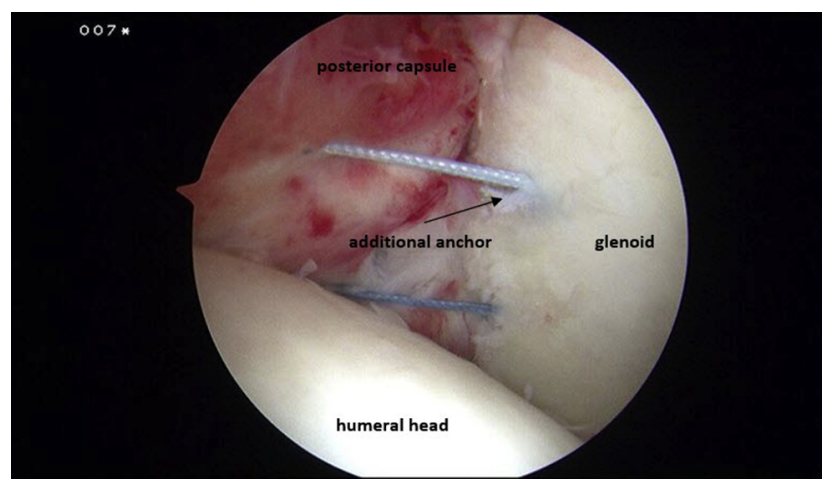

Fig 3. Arthroscopic view of the right shoulder in the beach chair position with a $30^{\circ}$ arthroscope in the anterosuperior portal. An additional biocomposite suture anchor (Arthrex) is placed on the posterior glenoid rim, and a retrograde suture retriever (IDEAL suture passer) is used to retrieve suture limbs through both the medial and lateral aspects of the capsular split. This suture is then tied in a mattress fashion.

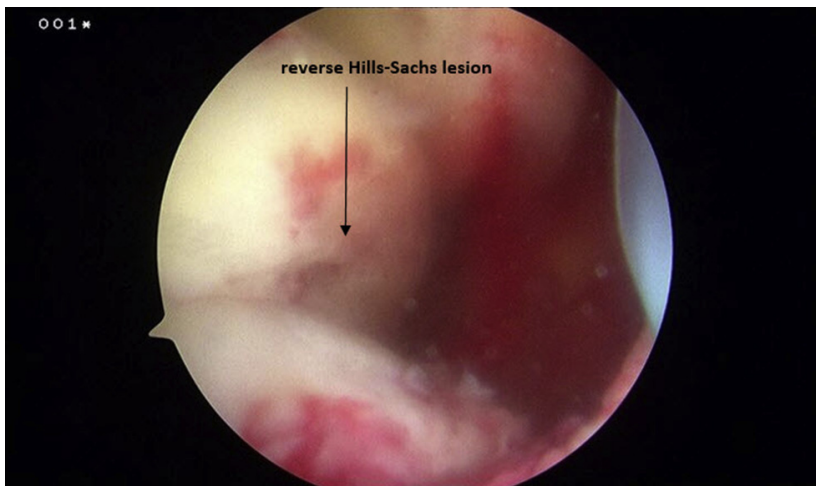

Fig 4. Reverse Hill-Sachs lesion of the right shoulder as viewed with a $30^{\circ}$ arthroscope through the anterosuperior portal with the patient in the beach chair position.

that mattress sutures be tied first, followed by the simple sutures. Following completion of arthroscopic knot tying, the subscapularis tendon is visualized through the anterosuperior portal advanced and secured into the reverse Hill-Sachs defect (Fig 7). The number and location of anchors can be modified as necessary depending on the size and orientation of the reverse Hill-Sachs lesion.

Rehabilitation following arthroscopic posterior stabilization and reverse remplissage is very similar to our standard postoperative protocol for posterior Bankart repairs. Patients are kept in a shoulder immobilizer in slight external rotation for 4 weeks postoperatively. The arm is maintained just posterior to the patient's trunk to minimize stress on the repair. Gentle elbow, wrist, and hand range-of-motion exercises are allowed during this time. After 4 weeks, patients begin active assisted range of motion to include forward elevation in the scapular plane and external rotation with the arm at the side. Internal rotation behind the patient's back is also

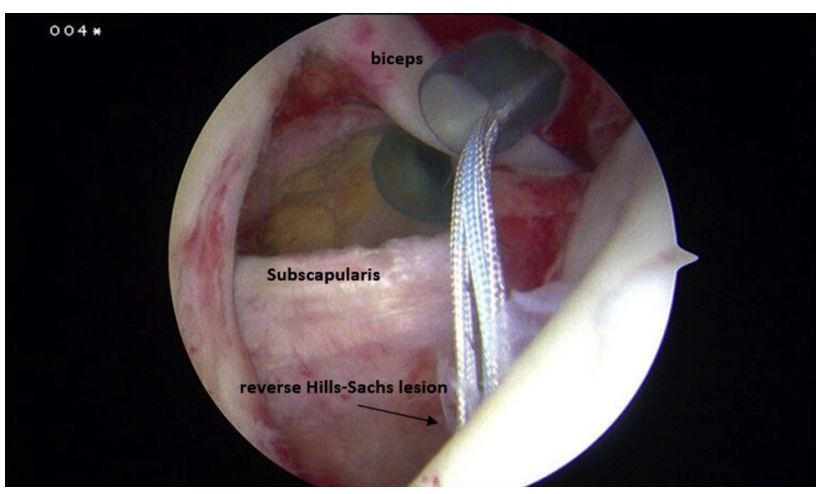

Fig 5. Arthroscopic view of the right shoulder in the beach chair position with a $30^{\circ}$ arthroscope in the posterior portal. A 5.5-mm triple-loaded biocomposite suture anchor (Smith \& Nephew) has been placed within the reverse Hill-Sachs lesion through the anteroinferior portal. 


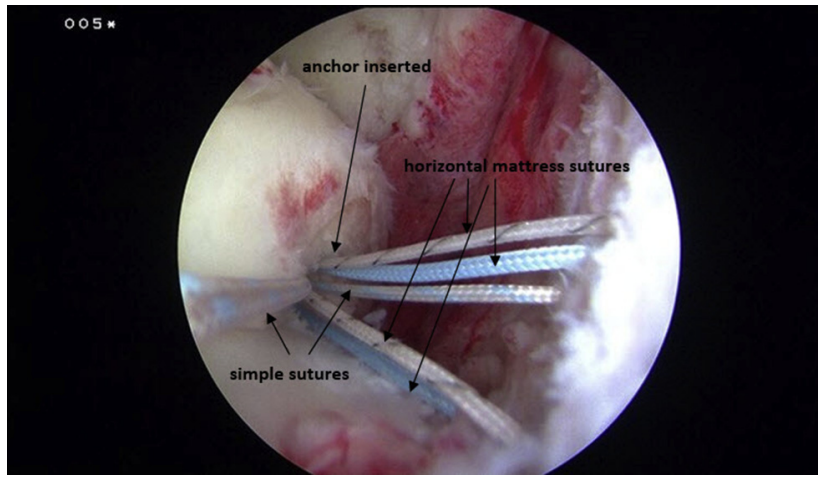

Fig 6. Arthroscopic view of the right shoulder in the beach chair position with a $30^{\circ}$ arthroscope in the anterosuperior portal. The sutures are passed through the subscapularis tendon using a retrograde suture retriever. Both limbs of the blue suture and black-striped suture have been passed in a horizontal mattress configuration, and 1 limb of the bluestriped suture has been passed inferior to the horizontal mattress to create a rip-stop construct once the sutures are tied.

initiated, but internal rotation with the arm elevated is prohibited at this time. Limited strengthening exercises are also initiated. At 8 weeks postoperatively, patients progress to more advanced rehabilitation with unrestricted range of motion and comprehensive shoulder and periscapular strengthening. Sport-specific training typically begins 3 to 4 months postoperatively, and return to collision sports and unrestricted weight lifting is usually allowed after 6 months.

\section{Discussion}

Posterior shoulder dislocations comprise $2 \%$ to $5 \%$ of glenohumeral dislocations. Locked posterior dislocations, while much less common, that are not recognized and appropriately managed may result in progressive joint destruction. ${ }^{3,6}$ Studies have shown that the diagnosis of locked posterior dislocation is missed or delayed in up to $79 \%$ of patients. ${ }^{6,11,12}$ Many of these cases of traumatic, posterior locked dislocations are reported in patient populations that include contact athletes, but they can also result from seizures and electrocution. $^{6,8}$ Saupe et al. showed that $86 \%$ of patients who sustained a traumatic posterior shoulder dislocation were found to have a reverse Hill-Sachs lesion. ${ }^{13}$ A separate study showed that a reverse HillSachs lesion greater than $1.5 \mathrm{~cm}^{3}$ was an independent risk factor for recurrent posterior dislocation and that $17.7 \%$ of such patients developed recurrent posterior shoulder dislocations within the first year following the initial posterior dislocation. ${ }^{14}$ As a consequence, it is vital that the treating physicians have a comprehensive understanding of the surgical techniques available to manage reverse Hill-Sachs lesions and accompanying posterior capsulolabral pathology. ${ }^{15}$
Open surgical techniques designed to directly treat these reverse Hill-Sachs lesions include transferring the lesser tuberosity or the subscapularis tendon into the bony impression fracture, filling the defect with autograft or allograft bone, humeral rotational osteotomies, and humeral head arthroplasty. ${ }^{6-8}$ These techniques are highly invasive, sometimes requiring both an open posterior and an additional open anterior approach, and may not be ideally suited for young, athletic individuals such as the patient undergoing operative intervention in this case example.

As arthroscopic treatment of posterior instability has evolved, multiple techniques have been proposed to arthroscopically address reverse Hill-Sachs lesions. Arthroscopic mobilization of the subscapularis tendon to fill the humeral head defect was originally described by Krackhardt et al. in 2006. ${ }^{9}$ A different technique to fill this defect that transferred the middle glenohumeral ligament was described by Duey and Burkhart in $2013 .{ }^{2}$ Additionally, Lavender et al. ${ }^{16}$ described a technique for arthroscopic reverse remplissage using multiple suture anchors. Despite the paucity of outcomes data regarding such procedures, there are many potential advantages of arthroscopic techniques. Most importantly, arthroscopic intervention facilitates treatment of all contributory lesions using a minimally invasive approach and allows for anatomic specific repairs based on the identified pathology. ${ }^{10}$ Nevertheless, certain limitations exist regarding our technique. This technique can reliably provide stability for posterior capsulolabral tears and smaller posterior bony Bankart lesions but may not be adequate for larger bony glenoid defects or excessive glenoid retroversion. Substantial posterior glenoid bone loss may require bony augmentation to achieve joint stability. Likewise, subsets with extremely large reverse HillsSachs lesions may be better treated with an open procedure such as a lesser tuberosity transfer, bone grafting,

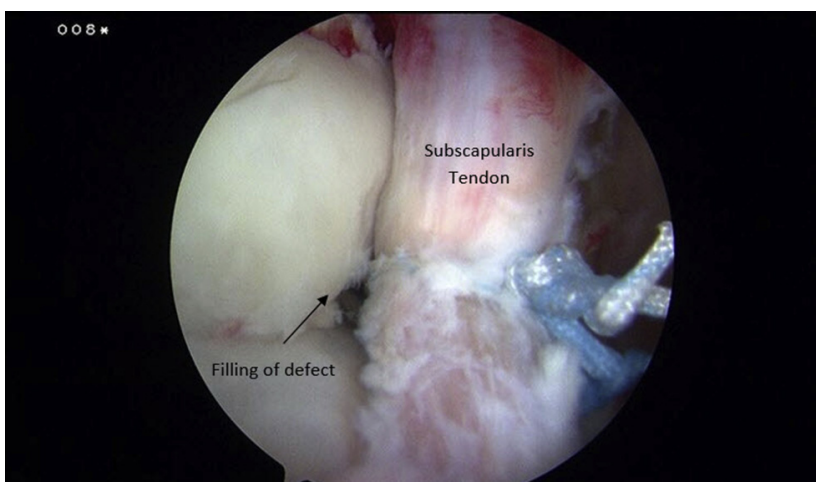

Fig 7. Arthroscopic view of the right shoulder in the beach chair position with a $30^{\circ}$ arthroscope in the anterosuperior portal. The subscapularis tendon has been reduced and compressed into the reverse Hill-Sachs defect as the horizontal mattress suture limbs, followed by the simple suture limbs, have been tied using an arthroscopic knot pusher. 
Table 2. Surgical Pearls and Pitfalls of the Arthroscopic Reverse Remplissage Technique

Surgical Pearls and Pitfalls

Paramount to assess and/or address both anterior and posterior pathology.

Perform thorough assessment and quantification of any glenoid bone loss.

Evaluate for engagement of the reverse Hills-Sachs lesion on the posterior glenoid.

Ensure sufficient posterior capsulorrhaphy is performed to adequately reduce posterior and inferior capsular volume.

Excision of central rotator interval tissue is essential for proper access and visualization of the subscapularis.

Use an arthroscopic shaver to adequately prepare the articular impaction of the reverse Hills-Sachs for reception of the subscapularis tendon.

or even arthroplasty. Lastly, our technique is likely to be more successful in the acute and subacute clinical scenarios. Patients with a chronically locked posterior shoulder dislocation would likely achieve better outcomes with an open surgical option (Tables 1 and 2). In conclusion, the surgical technique presented here to advance the subscapularis into the reverse Hill-Sachs defect uses equipment, portals, and suture management methods that are very familiar to the arthroscopic shoulder surgeon and can be reliably reproduced when indicated.

\section{References}

1. Khira YM, Salama AM. Treatment of locked posterior shoulder dislocation with bone defect. Orthopedics 2017;40(3).

2. Duey RE, Burkhart SS. Arthroscopic treatment of a reverse Hill-Sachs lesion. Arthrosc Tech 2013;2:e155-e159.

3. Martetschläger F, Padalecki JR, Millett PJ. Modified arthroscopic McLaughlin procedure for treatment of posterior instability of the shoulder with an associated reverse Hill-Sachs lesion. Knee Surg Sports Traumatol Arthrosc 2013:21:1642-1646.
4. Provencher MT, Frank RM, LeClere LE, et al. The HillSachs lesion: Diagnosis, classification, and management. J Am Acad Orthop Surg 2012;20:242-252.

5. Castagna A, Conti M, Garofalo R. Soft tissue-based techniques for treatment of posterior shoulder instability. Obere Extrem 2017;12(2):82-89.

6. Rouleau DM, Herbert-Davies J, Robinson CM. Acute traumatic posterior shoulder dislocation. J Am Acad Orthop Surg 2014;22:145-152.

7. Millett PJ, Clavert P, Hatch GFR, Warner JJ. Recurrent posterior shoulder instability. J Am Acad Ortho Surg 2006; 14:464-476.

8. Cicak N. Posterior dislocation of the shoulder. J Bone Joint Surg Br 2004;86:324-332.

9. Krackhardt T, Schewe B, Albrecht D, Weise K. Arthroscopic fixation of the subscapularis tendon in the reverse Hill-Sachs lesion for traumatic unidirectional posterior dislocation of the shoulder. Arthroscopy 2006;22:227.e1227.e6.

10. Savoie FH, Holt MS, Field LD, Ramsey JR. Arthroscopic management of posterior instability: Evolution of technique and results. Arthroscopy 2008;24:389-396.

11. Hatzis N, Kaar TK, Wirth MA, Rockwood CA. The often overlooked posterior dislocation of the shoulder. Tex Med 2001;97(11):62-67.

12. Hawkins RJ, Neer CS, Pianta RM, Mendoza FX. Locked posterior dislocation of the shoulder. J Bone Joint Surg Am 1987;61 (1):9-18.

13. Saupe N, White LM, Bleakney R, et al. Acute traumatic posterior shoulder dislocation: MR findings. Radiology 2008;248:185-193.

14. Robinson CM, Seah M, Akhtar MA. The epidemiology, risk of recurrence, and functional outcome after an acute traumatic posterior dislocation of the shoulder. J Bone Joint Surg Am 201 1;93:1605-1613.

15. Frank RM, Romeo AA, Provencher MT. Posterior glenohumeral instability: Evidence-based treatment. J Am Acad Ortho Surg 2017;25:610-623.

16. Lavender CD, Hanzlik SR, Pearson SE, Caldwell PE. Arthroscopic reverse remplissage for posterior instability. Arthrosc Tech 2016;5:e43-e47. 